\title{
Rabl8 Regulates Proliferation, Invasion and Cisplatin Sensitivity Through STAT3 Signaling in Head and Neck Squamous Cell Carcinoma
}

This article was published in the following Dove Press journal: OncoTargets and Therapy

\author{
$\mathrm{Xu} \mathrm{Ji}$ \\ Xing Guo' \\ Yan Wang' \\ Xiaotian $\mathrm{Li}^{1}$ \\ Hong $\mathrm{Li}^{2}$ \\ 'Department of Otolaryngology, The \\ First Affiliated Hospital of China Medical \\ University, Shenyang, People's Republic of \\ China; ${ }^{2}$ Department of Otolaryngology, \\ The Fourth Affiliated Hospital of China \\ Medical University, Shenyang, People's \\ Republic of China
}

Correspondence: $\mathrm{Xu} \mathrm{Ji}$

Department of Otolaryngology, The First Affiliated Hospital of China Medical

University

Email cmujixu@163.com

Hong Li

Department of Otolaryngology, The

Fourth Hospital of China Medical

University, Shenyang, People's Republic of

China

Email lihongcmu4@I63.com
Introduction: The clinical significance, biological roles and potential mechanism of Rab18 remain unknown in most human cancers, including head and neck squamous cell carcinoma (HNSCC).

Methods: We used immunohistochemistry to examine Rab18 protein expression in 112 cases of HNSCC specimens. We overexpressed and knockdown Rab18 in FaDu and Detroit562 cancer cell lines. Biological roles and mechanisms of Rab18 were examined using MTT, colony formation, Matrigel invasion assay, Western blotting, Annexin V and JC1 staining.

Results: Rab18 was upregulated in 45/112 (40.2\%) cases of HNSCC tissues, which correlated with advanced $\mathrm{T}$ classification, positive nodal metastasis and tumor node metastasis (TNM) stage. The Oncomine and The Cancer Genome Atlas (TCGA) analyses indicated that Rab18 was elevated in human HNSCC tissues and correlated with poor patient survival. Functionally, Rab18 overexpression increased growth rate, colony numbers, cell cycle progression and invading ability in FaDu cells. Rab18 downregulated cisplatin-induced apoptosis and upregulated the mitochondrial membrane potential $(\Delta \psi \mathrm{m})$. Western blot revealed that Rab18 overexpression induced epithelial-to-mesenchymal transition, with downregulation of E-cadherin and upregulation of N-cadherin, Vimentin and Twist. Rab18 overexpression also upregulated Survivin protein and Rab18 knockdown showed the opposite effects on these proteins. Treatment of STAT3 inhibitor, SH-4-54, inhibited cell invasion, increased E-cadherin and downregulated N-cadherin, Twist and Survivin. SH-4-54 also abolished the effects of BCAT1 on these proteins, as well as cell invasion.

Conclusion: In summary, our data showed that Rab18 was overexpressed in human HNSCC and functioned as an oncoprotein. Rab18 regulated HNSCC cell proliferation, invasion and cisplatin sensitivity through STAT3 signaling in HNSCC.

Keywords: Rab18, STAT3, proliferation, E-cadherin, invasion

\section{Introduction}

Head and neck squamous cell carcinoma (HNSCC) is the sixth most common malignancy worldwide and its incidence is increasing. ${ }^{1,2}$ Despite aggressive treatment strategies including surgery, chemotherapy and radiotherapy, the survival rate of HNSCC has remained poor during past decades. Development of invasion and metastasis is important causes of cancer-related mortality in HNSCC. Thus, it is of great importance to identify novel bio-markers, especially those associated with cell invasiveness. 
Rab18 is a member of the Rab GTPase family which regulates vesicular transportation during exocytic and endocytic processes. ${ }^{3}$ Recent studies indicated potential association between Rab family proteins and carcinogenesis. $^{3-5}$ Rab25 was reported to be downregulated in HNSCC and suppressed tumor migration and metastasis. ${ }^{6}$ Rab18 has been reported to be involved in the lipogenesis of 3T3-L1 adipocytes. ${ }^{7}$ Loss of function mutations of Rab18 caused Warburg Micro syndrome. ${ }^{8}$ Rab18 acts as a novel tumor antigen in the screening of childhood medulloblastoma DNA libraries. ${ }^{9}$ Rab18 serves as a promoter of proliferation in hepatoma cell lines. ${ }^{10}$ Rab18 overexpression promotes proliferation and metastasis in hepatocellular carcinoma, possibly through regulation of cell cycle proteins and epithelial-mesenchymal transition. ${ }^{11}$ In addition, Rab18 promotes proliferation and chemoresistance through regulation of mitochondrial function in human gastric cancer. ${ }^{12}$ These data suggest that Rab18 is a potential oncoprotein during cancer development. However, its clinical significance and the potential underlying mechanism remain unclear in HNSCC.

The aim of the present study was, therefore, to determine the clinical significance of Rab18 in HNSCC. Moreover, we also identified its biological roles in HNSCC cells. Our findings provided new evidence that Rab18 protein contributes to the carcinogenesis and progression of HNSCC.

\section{Patients and Methods}

\section{Patients and Samples}

This study protocol was approved by the Institutional Reviewer Board of China Medical University (approval number: 2014-015). HNSCC specimens and adjacent normal tissues were obtained from patients who underwent surgical resection in First affiliated Hospital of China Medical University between 2010 and 2015. Written informed consents were obtained from the patients. The study was conducted in accordance with the Declaration of Helsinki.

\section{Immunohistochemistry}

4- $\mu \mathrm{m}$ tissue sections were prepared and $\mathrm{H}_{2} \mathrm{O}_{2}$ was used to block the endogenous peroxidase. Rab18 polyclonal antibody (1:250 dilution; Proteintech, USA) was used to incubate sections overnight at $4{ }^{\circ} \mathrm{C}$. The Elivision plus kit obtained from Maixin (Fuzhou, China) was used for immunohistochemistry reaction. Counterstaining was performed using hematoxylin.

Expression of Rab18 was evaluated by a pathologist. Immunohistochemistry of Rab18 was graded using a semiquantitative scale by assessing both intensity and percentage of stained cells. Cytoplasmic immunopositivity was assumed to be positive Rab18 staining. Rab18 intensity was scored as 0(negative/weak), 1 (moderate), 2 (strong). Percentage was scored as 1- $1-25 \%, 2-26-50 \%, 3-$ $51-75 \%$ and $4-76-100 \%$. 2 scores were multiplied to a final score $(0-8)$. Tumors were finally determined as Rab18 low expression when score $<4$, tumor sample scored $\geq 4$ were considered as Rab18 high expression.

\section{Cell Culture}

HNSCC cell lines FaDu, KB, Detroit562 were obtained from Shanghai Cell Bank, Chinese Academy of Sciences. The cell lines were cultured with RPMI-1640 (Gibco, USA) containing fetal bovine serum (FBS).

pCMV6-Rab18 plasmid was obtained from Origene (Origene, Rockville, USA). Lipofectamine 3000 reagent was used for plasmid transfection (Invitrogen, USA). In brief, Lipofectamine 3000 was mixed with and DNA for 15 minutes in MEM media without FBS, then the mixture was added drop by drop into the culture media.

Control and Rab18-specific siRNA were obtained from Dharmacon (siRNA1: UAAACAUGCUAGUUGGAAA; siRNA2: UGCACAGGGUGUUAUAUUA). SiRNAs were transfected using Dharmafectl reagent (Dharmacon, USA). Dharmafect1 was mixed with and siRNA for 15-20 minutes in MEM media without FBS, then the mixture was added drop by drop into the culture media.

\section{Quantitative Real-Time PCR (SYBR Green Method)}

RNA was extracted using RNAiso plus reagent (TAKARA, Dalian, China). Reverse transcription was performed using RT kit from TAKARA (Dalian, China). Real-time PCR was performed using SYBR Green master mix (TAKARA, Dalian, China). $\beta$-actin was used as control and relative expression of target genes was calculated using the $2^{-\Delta \Delta \mathrm{Ct}}$ method. The primer sequences are as follows: Rab18 forward, 5' CAATGTGCCTTTGAAGAA CTTGT 3', Rab18 reverse, 5' CTCCTTGGCCTTCTTC CCTG 3'. $\beta$-actin forward, 5' ATAGCACAGCCTGGAT AGCAACGTAC 3', $\beta$-actin reverse, 5' CACCTTCTACA 
ATGAGCTGCGTGTG $3^{\prime}$. The experiment was performed in triplicate.

\section{Western Blotting}

Protein was extracted from cell and separated using SASPAGE. Denatured protein was then transferred onto PVDF membrane. The membranes were incubated with antibodies against Rab18 (1:800, Proteintech, USA), E-cadherin, N-cadherin, Vimentin, Survivin, p-STAT3, STAT3, Twist (1:900, Cell Signaling, USA) and GAPDH (1:3000). After incubated with HRP-coupled antibodies (1:3000, Santa Cruz, USA), the membranes were visualized with HRP Substrate and images were captured by DNR imaging system.

\section{MTT and Colony Formation Assay}

1000 cells were seeded in 6-well plates and then cultured for about 2 weeks. The plate was fixed with paraformaldehyde (PFA). After washing with PBS, the plates were stained using Giemsa. For MTT assay, transfected cells were plated in a 96-well plates (2000 cells each well). $20 \mu \mathrm{L}$ of $5 \mathrm{mg} / \mathrm{mL}$ thiazolyl blue (MTT) solution was added to wells. The plates were incubated for 4 hours. Then, $150 \mu \mathrm{L}$ DMSO was used to dissolve the MTT crystal in the bottom of each well, which was measured at $490 \mathrm{~nm}$ wavelength. The experiment was performed in triplicate.

\section{Matrigel Invasion Assay}

A Cell invasion assay was performed using a Transwell chamber coated with $18 \mu \mathrm{L}$ Matrigel from BD Bioscience (dilution rate of 1:4). Cells were suspended and $100 \mu \mathrm{L}$ of serum-free medium and transferred to the upper chamber and incubated for 20 hours. Medium with 15\% FBS was added to the lower chamber. The non-invading cells in the upper chambers were removed using a cotton tip. Cells passing through the filter were fixed, stained with hematoxylin and counted using a microscope. The experiment was performed in triplicate.

\section{Cell Cycle Analysis}

Forty-eight hours after transfection, the cells were harvested and fixed using 1\% paraformaldehyde. The cells were then washed with PBS and stained in $5 \mathrm{mg} / \mathrm{mL}$ propidium iodide for 30 minutes at room temperature. Flow cytometry was performed using flow cytometer. The experiment was performed in triplicate.

\section{Annexin V/PI and Mitochondrial Membrane Potential}

To determine the apoptosis percentage, Annexin V/PI staining kit (BD bioscience) was used for cell staining. Then, cells with Annexin V/PI staining were analyzed with flow cytometer. For detection of mitochondrial membrane potential $(\Delta \psi \mathrm{m})$. JC-1 dye (Cell Signaling Technology) was used to stain cells for 30 minutes. After that cells were washed and analyzed using flow cytometer. The experiment was performed in triplicate.

\section{Statistical Analysis}

We adopted SPSS version 16 for statistical analysis. $\chi^{2}$ test was used to examine possible correlations between Rab18 expression and clinicopathological factors. Differences between transfection/control groups were compared using Student's $t$-test. $\mathrm{p}<0.05$ was considered as statistically significant.

\section{Results}

\section{Rabl8 Expression Is Elevated in HNSCC}

Rab18 expression was examined in 112 cases of HNSCC tissues and 20 cases of adjacent normal tissues using immunohistochemistry. We determined Rab18 protein levels in tongue tissues (Figure 1A), throat tissues (Figure 1B) and salivary gland tissues (Figure 1C). In normal tissues, Rab18 expression was weak in tongue tissues and throat mucosa. In the salivary gland, Rab18 showed negative staining. Rab18 protein mainly localized in the cytoplasm of tumor tissues. In the 112 HNSCC tissues examined, 40.2\% (45/112) showed high Rab18 levels (Figure 1D and F). The remaining cases showed weak staining or negative staining (Figure 1E). The median expression score of Rab18 was 3 in HNSCC and was 0 in normal tissues. We analyzed the association between Rab18 status and clinical features. As shown in Table 1, Rab18 high expression correlated with advanced TNM stage $(\mathrm{p}=0.0003)$, nodal metastasis $(\mathrm{p}=0.0027)$ and higher $\mathrm{T}$ stage $(\mathrm{p}=0.0123)$. No significance was observed between Rab18 and other clinical features (age, sex and tumor grade). We also determined the Rab18 mRNA expression of HNSCC tissues and normal tissues using data from Oncomine database. As shown in Figure 1G The Sengupta Head-neck of Oncomine showed that Rab18 mRNA is significantly higher in nasopharyngeal carcinoma tissues compared with normal nasopharyngeal tissues $(\mathrm{p}=0.029$ ). Furthermore, The Cancer Genome Atlas (TCGA) HNSCC cohort showed that the survival rate of 
A

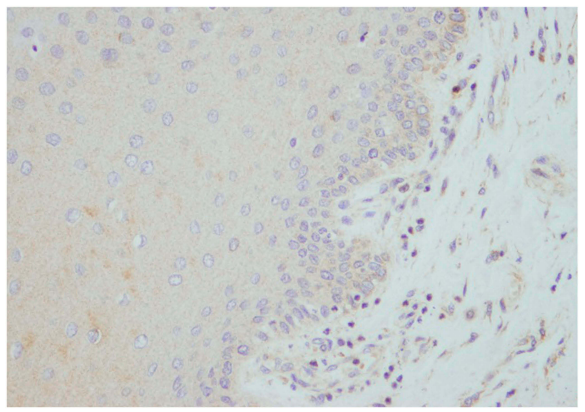

C

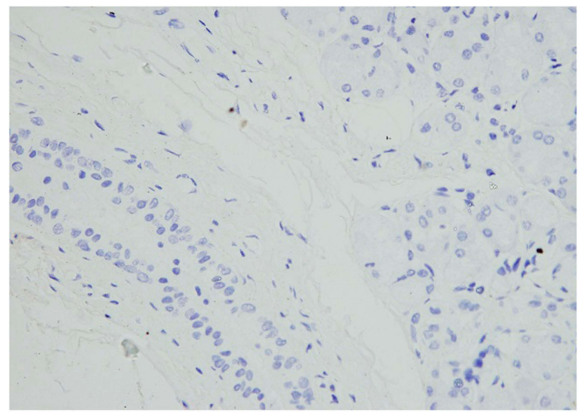

E

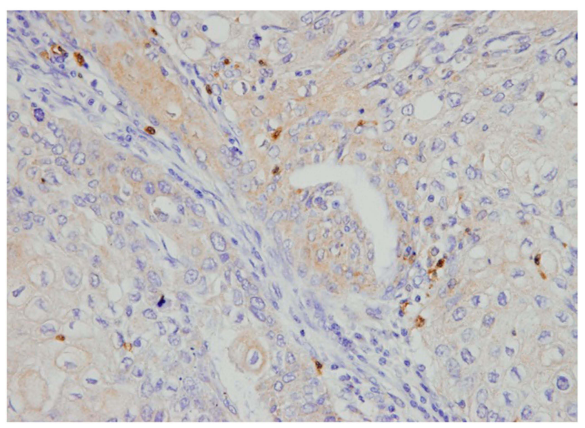

B

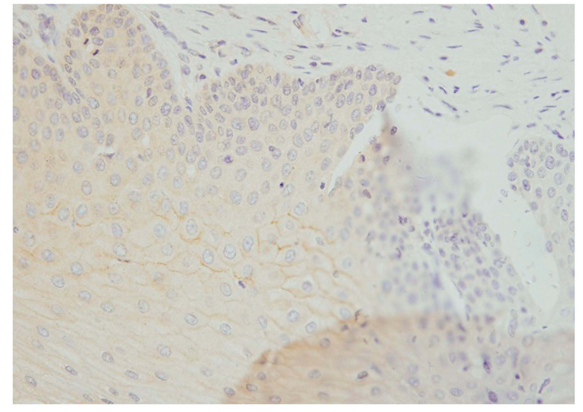

D

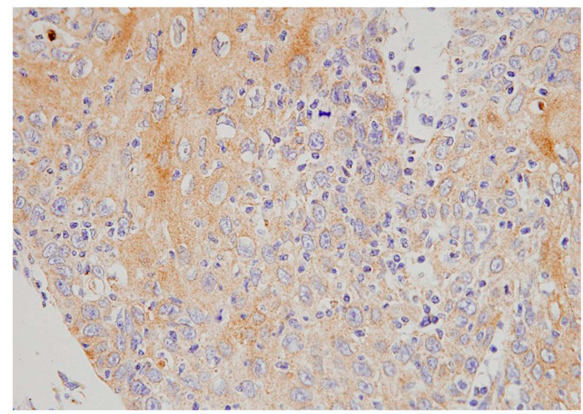

F

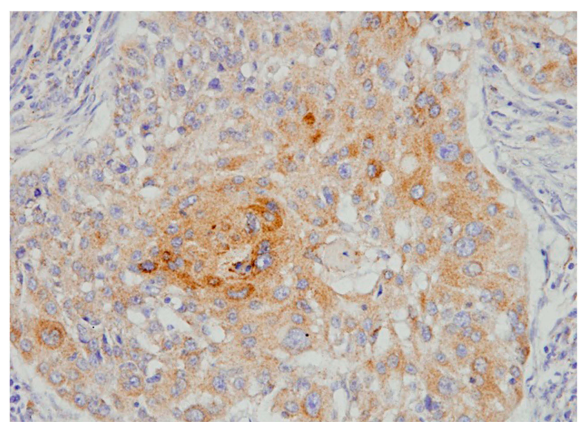

G

Sengupta Head-Neck Statistics of Oncomine

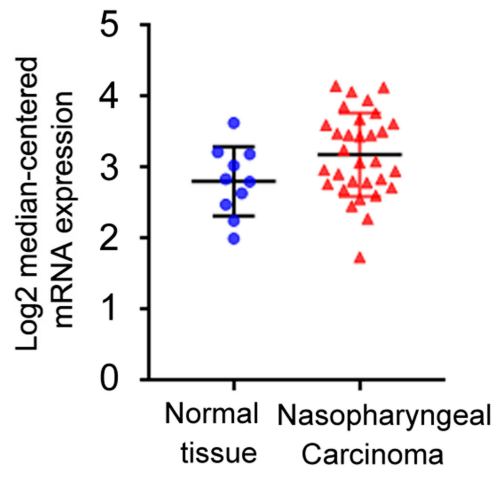

H

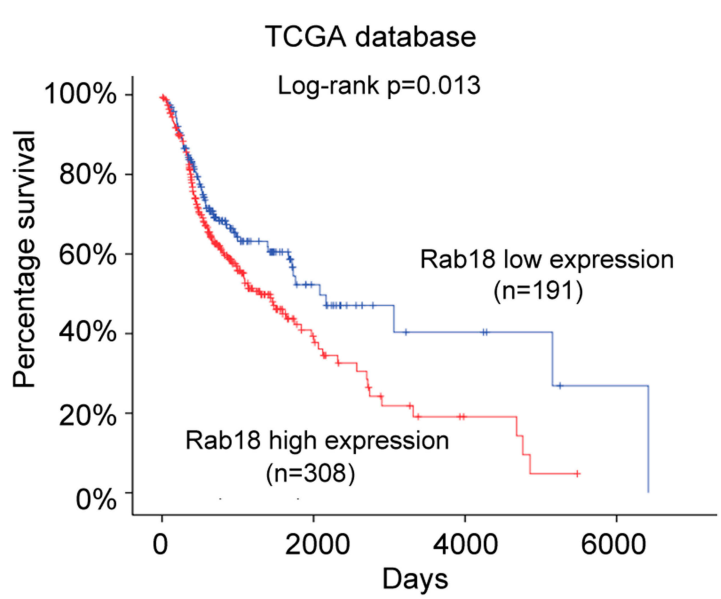

Figure I Expression of Rabl8 in HNSCC. (A) Negative staining of Rabl8 in normal tongue tissue (intensity score 0). (B) Weak staining of Rab/8 in normal throat cells (intensity score 0). (C) Negative staining of Rabl8 in salivary gland tissue (intensity score 0). (D) Moderate cytoplasmic Rabl8 expression in squamous cell carcinoma of lower lip (intensity score I). (E) Weak cytoplasmic staining of Rabl8 in squamous cell carcinoma of larynx (intensity score 0). (F) Strong Rabl8 cytoplasmic expression in squamous cell carcinoma of larynx (intensity score 2). (G) Rab 18 mRNA in Sengupta Head-neck of Oncomine database. Rab 18 mRNA is higher in nasopharyngeal carcinoma tissues compared with normal nasopharyngeal tissues $(p=0.029)$. $(\mathbf{H})$ Patient survival analysis of the Cancer Genome Atlas (TCGA) HNSCC cohort. Patients with low Rab 8 showed better prognosis. (Log-rank test, $\mathrm{p}=0.013$ ). Magnification: $400 \times$.

Abbreviation: HNSCC, non-small cell lung cancer. 
Table I Clinical Profile and Correlation Between the Clinicopathological Features and Rabl8 Expression in HNSCC

\begin{tabular}{|c|c|c|c|c|}
\hline Characteristics & Number of Patients & Rab|8 Low Expression & RabI8 High Expression & $P$ \\
\hline \multicolumn{5}{|l|}{ Age } \\
\hline$<60$ & 62 & 35 & 26 & 0.6728 \\
\hline$\geq 60$ & 50 & 31 & 19 & \\
\hline \multicolumn{5}{|l|}{ Gender } \\
\hline Male & 88 & 51 & 37 & 0.4403 \\
\hline Female & 24 & 18 & 8 & \\
\hline \multicolumn{5}{|l|}{ Differentiation } \\
\hline Well & 59 & 39 & 20 & 0.1526 \\
\hline Moderate-Poor & 53 & 28 & 25 & \\
\hline \multicolumn{5}{|l|}{ TNM Stage } \\
\hline $1+11$ & 72 & 52 & 20 & 0.0003 \\
\hline III+IV & 40 & 15 & 25 & \\
\hline \multicolumn{5}{|l|}{ Lymph Node Metastasis } \\
\hline Absent & 84 & 57 & 27 & 0.0027 \\
\hline Present & 28 & 10 & 18 & \\
\hline \multicolumn{5}{|l|}{ Tumor Stage } \\
\hline $\mathrm{TI}+\mathrm{T} 2$ & 90 & 59 & 31 & 0.0123 \\
\hline $\mathrm{T} 3+\mathrm{T} 4$ & 22 & 8 & 14 & \\
\hline
\end{tabular}

Abbreviations: HNSCC, non-small cell lung cancer; TNM, tumor-node-metastasis; T, tumor.

patients with low Rab18 mRNA was better than those with high Rab18 mRNA. (Log-rank test, $\mathrm{p}=0.013$, Figure $1 \mathrm{H}$ ). Collectively, these data indicated that Rab18 was upregulated and correlated with malignant features in human HNSCC.

\section{Rab I 8 Promotes Proliferation, Invasion and Cell Cycle Progression in HNSCC Cell Lines}

Protein and mRNA levels of Rab18 were examined in KB, $\mathrm{FaDu}$ and Detroit562 cell lines. Western blot and RT-qPCR indicated that Rab18 expression was relatively high in Detroit562 cell line while low in $\mathrm{FaDu}$ and $\mathrm{KB}$ cell lines (Figure 2A). To find out the biological function of Rab18 in HNSCC, Rab18 overexpression was conducted in the FaDu cell line and Rab18 siRNA knockdown was conducted in the Detroit562 cell line. The efficiencies of siRNA1 and plasmid transfection were validated using Western blot and RT-qPCR (Figure 2A). The knockdown effect of Rab18 siRNA2 was shown in the Supplementary Figure 1A. MTT assay showed that Rab18 overexpression increased proliferation rate while Rab18 siRNA decreased proliferation rate (Figure 2B). Colony formation showed that Rab18 overexpression increased colony numbers while Rab18 depletion decreased colony numbers (Figure 2C). Cell cycle analysis showed that Rab18 facilitated cell cycle transition in FaDu cell line, while Rab18 depletion blocked cell cycle transition in Detroit562 cell line with decreased $\mathrm{S}$ phase percentage (Figure 3A). Because Rab18 overexpression correlated with nodal metastasis, we evaluated the functional significance of Rab18 on invasion. Rab18 overexpression increased the invasion ability of $\mathrm{FaDu}$ cell line and Rab18 siRNA knockdown reduced the invading ability of Detroit562 cell line (Figure 3B).

\section{Rabl8 Downregulates Cisplatin Sensitivity and Maintains Mitochondrial Membrane Potential}

Next, we investigated the effect of Rab18 on cisplatin sensitivity in HNSCC cells. We used cisplatin $(2 \mu \mathrm{M})$ to treat HNSCC cells with Rab18 overexpression and depletion, and examined the inhibition rate using MTT assays. Rab18 overexpression decreased inhibition rate while Rab18 depletion increased inhibition rate (Figure 4A). The apoptosis rate was examined using AnnexinV/PI staining. As shown in Figure 4B, Rab18 overexpression reduced the levels of cisplatin-induced apoptosis after 24 
A
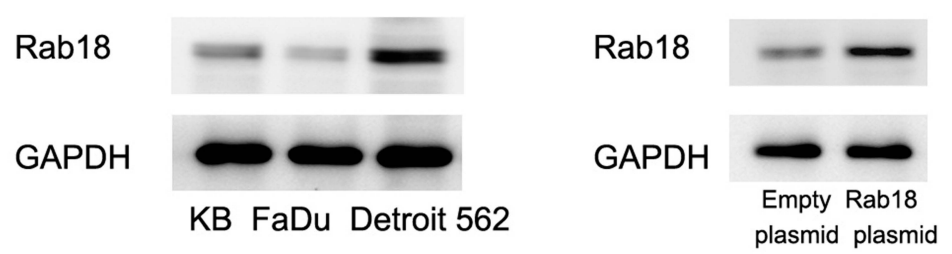

FaDu

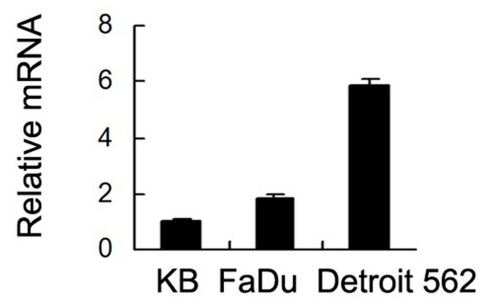

B

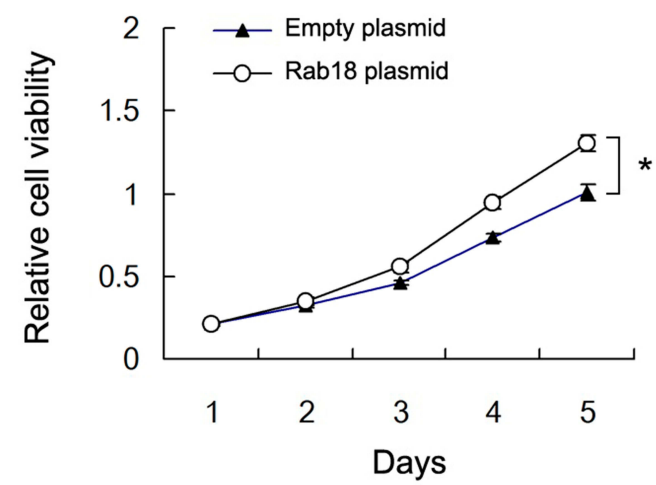

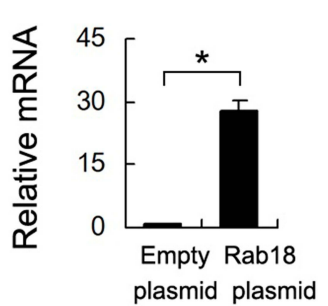

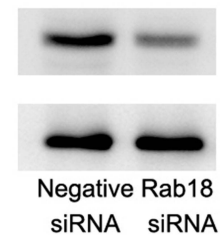

Detroit 562

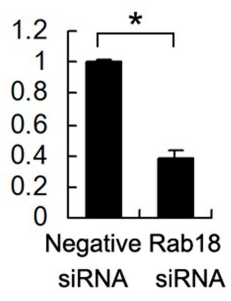

C

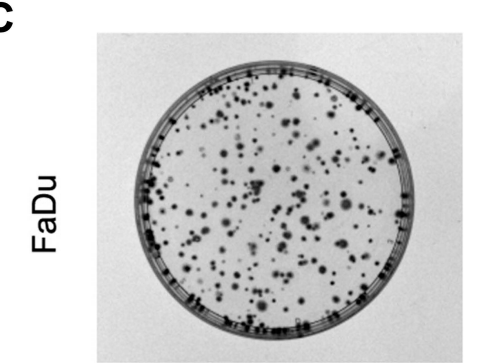

Empty plasmid

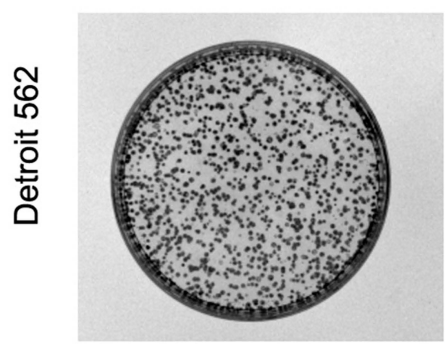

Negative siRNA

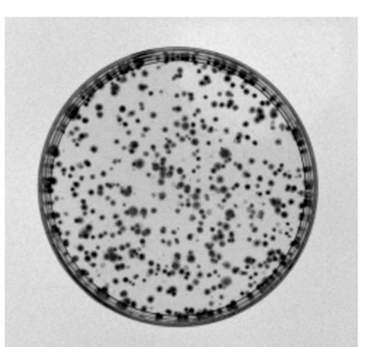

Rab18 plasmid

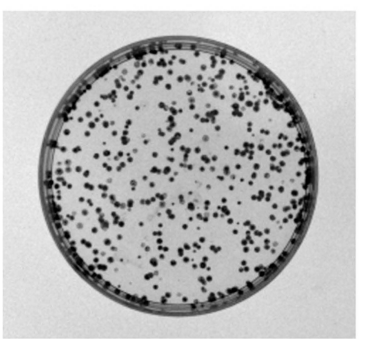

Rab18 siRNA
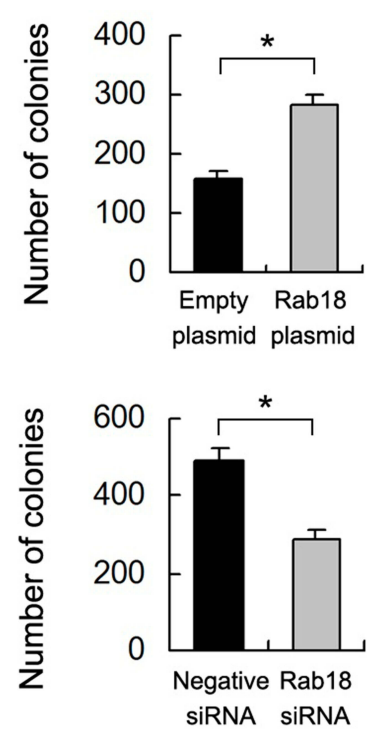

Figure 2 Rabl8 promotes proliferation and colony formation. (A) Endogenous expression of Rabl8 was examined in NHSCC cell lines (KB, FaDu, Detroit562). Western blot and RT-qPCR analysis showed that PCMV6-Rab 18 plasmid markedly increases its levels in FaDu cells. Rab I8 siRNA downregulated its expression in Detroit562 cells. (B) MTT assay showed that Rabl8 overexpression in FaDu cells significantly increased the proliferation rate while Rabl8 depletion decreased proliferation rate. (C) Rabl8 overexpression in FaDu cells promoted the colony formation ability while Rabl8 depletion inhibited colony formation ability. ${ }^{*} \mathrm{p}<0.05$.

Abbreviations: MTT, (3-(4,5-dimethylthiazol-2-yl)-2,5-diphenyltetrazolium bromide); HNSCC, non-small cell lung cancer; siRNA, small interfering RNA. 
A

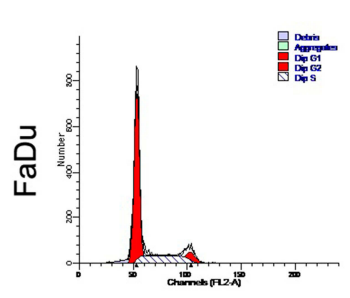

Empty plasmid

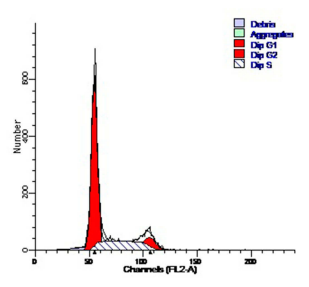

Rab18 plasmid

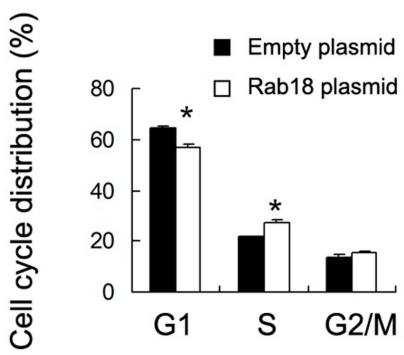

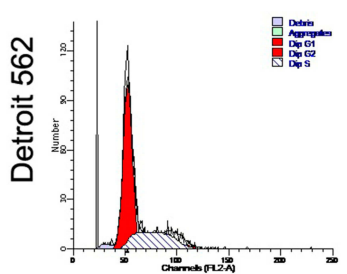

Negative siRNA

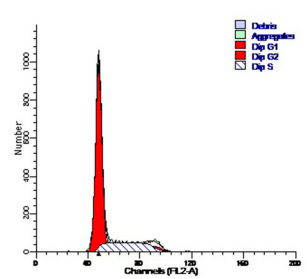

Rab18 siRNA
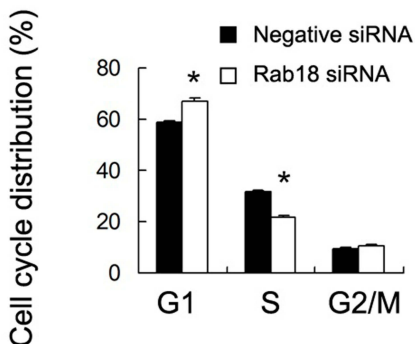

B
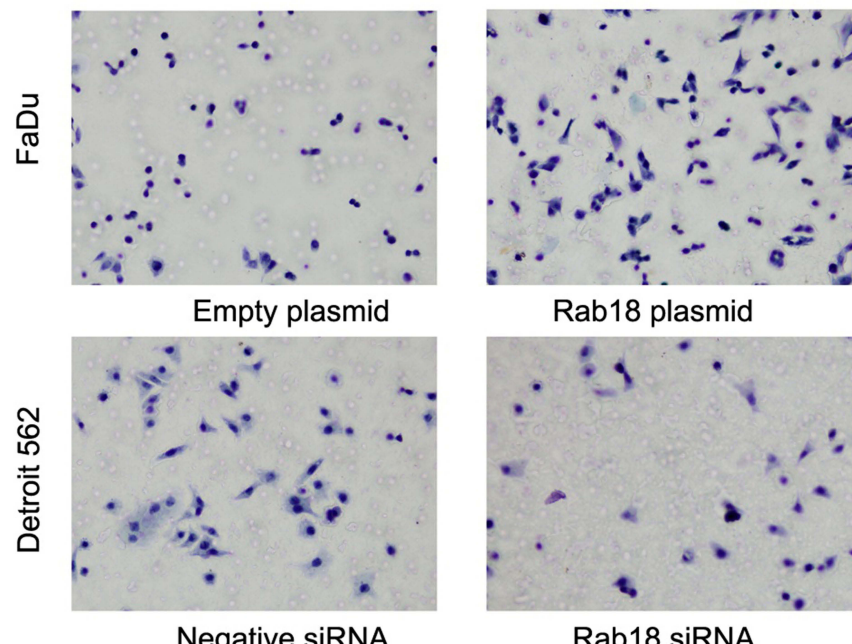

Rab18 plasmid

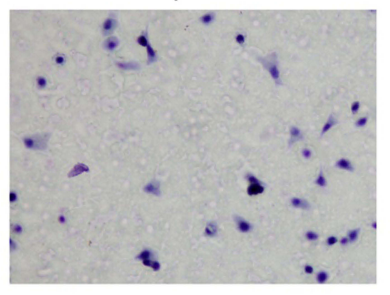

Rab18 siRNA
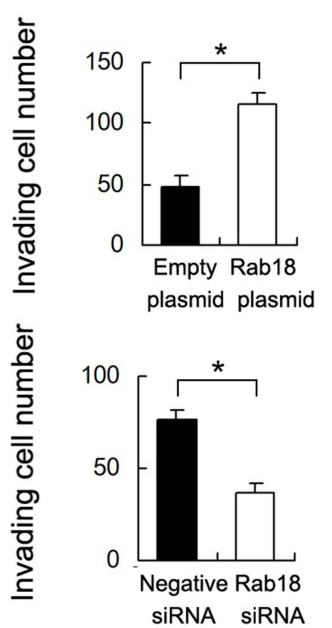

Figure 3 Rabl 8 promotes cell cycle progression and invasion. (A) Cell cycle analysis showed that Rabl8 transfection increased percentage of $\mathrm{S}$ phase and decreased the percentage of GI phase. Rabl8 depletion exhibited the opposite effects. (B) Rabl8 overexpression in FaDu cells greatly promoted cell invasion while Rabl8 depletion inhibited invading ability of Detroit562 cells. $* p<0.05$.

Abbreviations: $\mathrm{S}$ phase, synthesis phase; GI phase, Gap I phase; G2/M phase, Gap 2/Mitotic phase; FL2-A, fluorescence parameter 2-A; siRNA, small interfering RNA.

hours treatment, while Rab18 knockdown increased cisplatin-induced apoptosis, indicating Rab18 conferred resistance to cisplatin treatment in HNSCC cells. In addition, Rab18 also conferred resistance to 5-fluorouracil (5-Fu, $2 \mu \mathrm{g} / \mathrm{mL}$ ) in HNSCC cells (Supplementary Figure 1B).

Maintaining normal mitochondrial membrane potential $(\Delta \psi \mathrm{m})$ is important during cell survival when treated with chemotherapeutic drugs. We examined whether Rab18 regulated $\Delta \psi \mathrm{m}$ using JC-1 staining, which normally exhibits intense red fluorescence while turns green when $\Delta \psi \mathrm{m}$ is decreased. As shown in Figure 4C, Rab18 reduced the percentage of green staining while Rab18 depletion increased the percentage of green staining in cells treated with cisplatin. These findings suggested that Rab18 maintained a normal $\Delta \psi \mathrm{m}$ in cancer cells during cisplatin treatment. 
A

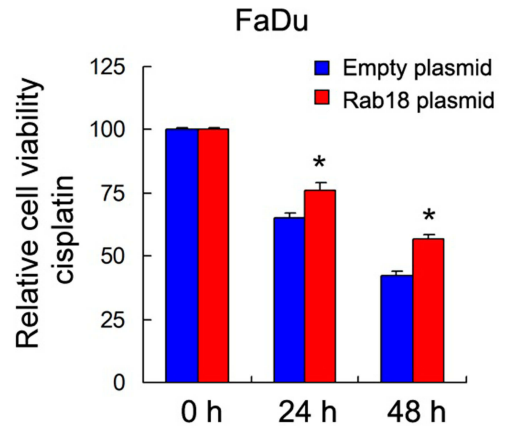

Detroit 562

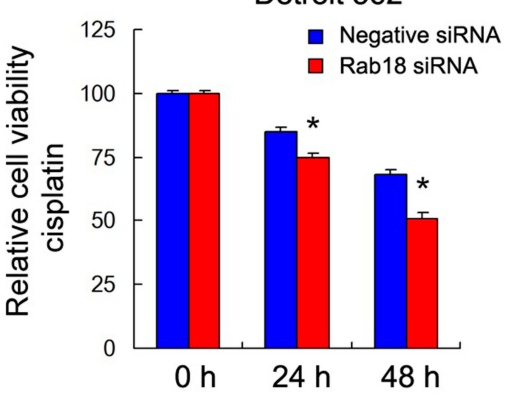

B
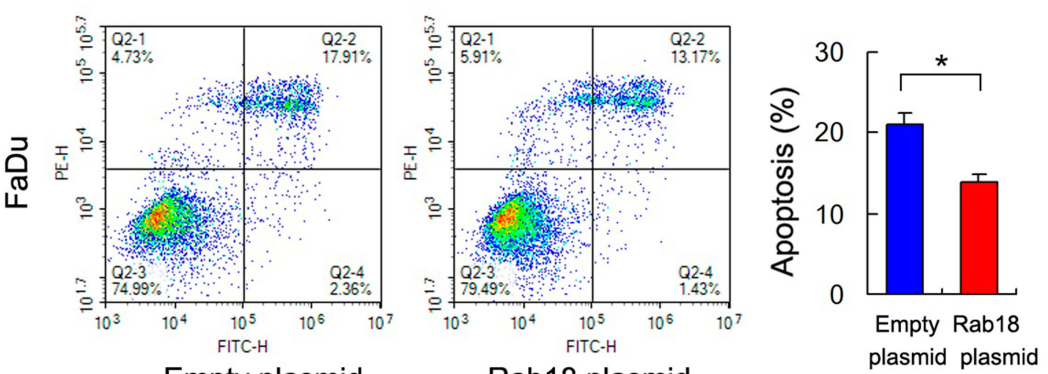

Empty plasmid

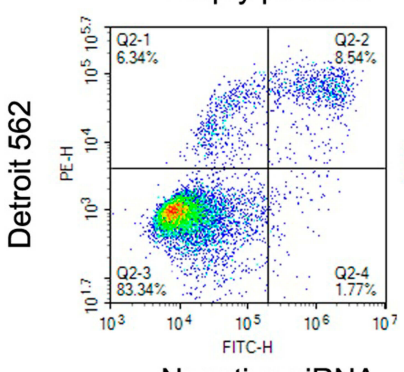

Rab18 plasmid
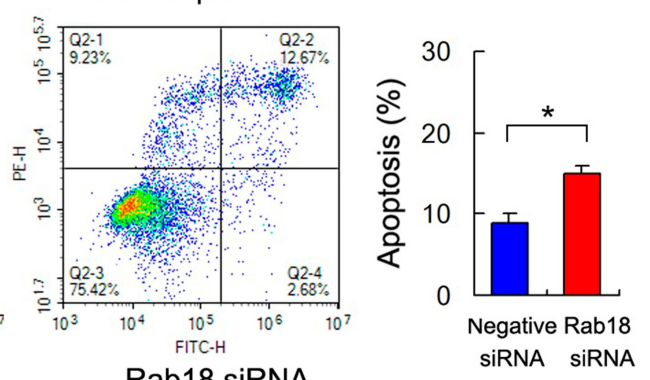

C
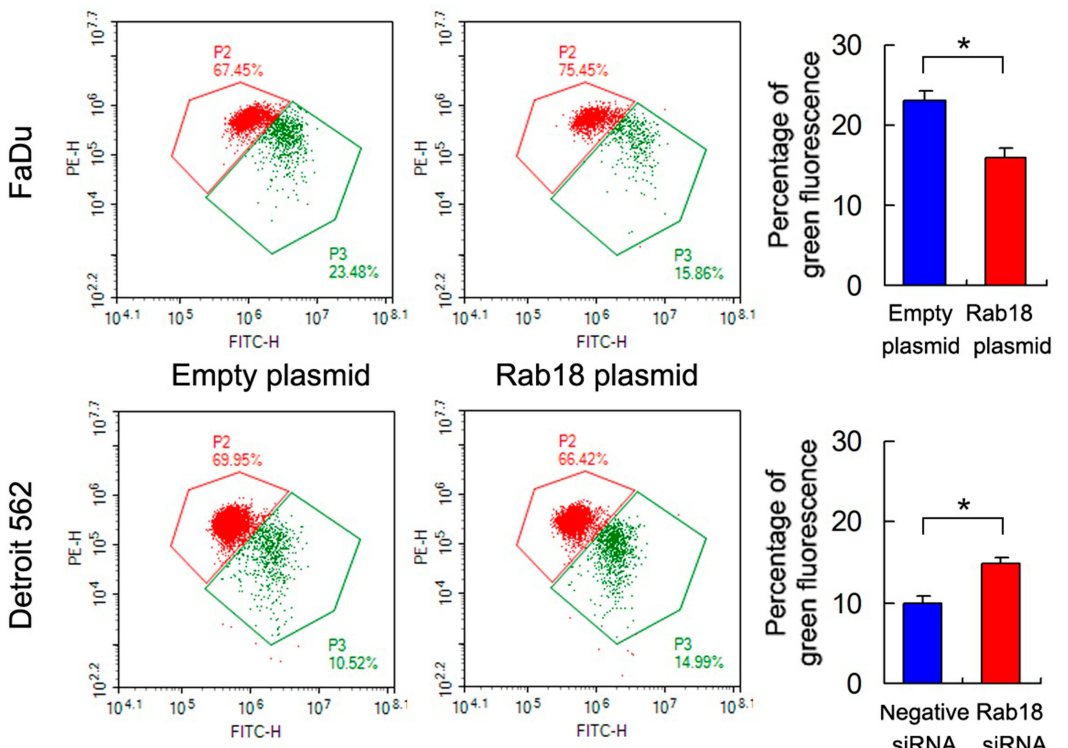

Negative siRNA
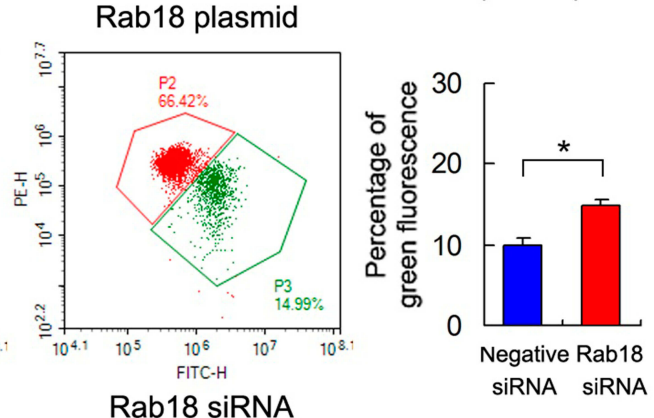

Figure 4 Rabl8 regulates cisplatin sensitivity and mitochondrial membrane potential. (A) MTT demonstrated that Rab 18 overexpression decreased inhibition rate while Rab I8 depletion increased inhibition rate in cancer cells treated with $2 \mu \mathrm{mol} / \mathrm{L}$ concentration of cisplatin. (B) Annexin V/PI analysis showed that Rab 18 overexpression decreased apoptosis while Rabl8 depletion increased apoptosis rate in gastric cancer cells treated with cisplatin. (C) Flow cytometry showed that Rabl8 overexpression increased mitochondrial membrane potential with increased JC-I red/green ratio, while Rab 18 depletion decreased mitochondrial membrane potential with decreased JC-I red/green ratio. * $<0.05$. Abbreviations: MTT, (3-(4,5-dimethylthiazol-2-yl)-2,5-diphenyltetrazolium bromide); PI, propidium iodide; siRNA, small interfering RNA; FITC-H, fluorescein isothiocyanate-H; PE-H, phycoerythrin-H. 


\section{Rabl 8 Regulates E-Cadherin, Twist, Survivin and STAT3 Signaling in HNSCC Cells}

To further elucidate the underlying mechanisms, we determined changes of several related proteins and signaling pathways. Our results showed that Rab18 overexpression inhibited E-cadherin expression and upregulated $\mathrm{N}$-cadherin, Vimentin, Twist and Survivin protein expressions in FaDu cell line. In addition, Rab18 overexpression increased phosphorylation of STAT3. In contrast, Rab18 depletion increased E-cadherin and decreased N-cadherin, Vimentin, Twist, Survivin and p-STAT3 protein levels in the Detroit562 cell line (Figure 5). Rab18 slightly increased expression of total STAT3 (STAT3 relative expression 1:1.32, calculated by ImageJ).

\section{Rab I8 Regulates E-Cadherin, Survivin and Invasion Through STAT3 Signaling Pathway} Activation of STAT3 signaling has been reported to induce the expressions of Survivin and the EMT. We tried to validate the association between STAT3 and Rab18 by using SH-4-54, a STAT3 inhibitor. SH-4-54 blocked phosphorylation of STAT3 (Figure 6A). Western blot showed that SH-4-54 treatment upregulated E-cadherin protein and downregulated protein levels of Twist, N-cadherin and
Survivin. In addition, in cells treated with SH-4-54, the effects of Rab18 on E-cadherin, Twist, N-cadherin and Survivin were not significant. In addition, SH-4-54 treatment reduced cell invasiveness and reduced the invasion promoting role of Rab18 (Figure 6B). STAT3 inhibition could abolish the effects of Rab18 on proliferation and drug resistance (Supplementary Figure 1C and D). The above data indicated STAT3 played an important role in the biological effects induced by Rab18.

\section{Discussion}

Recent evidences suggested Rab18 as a cancer-related protein. Rab18 overexpression has been reported in gastric cancer, ${ }^{12}$ hepatocellular carcinoma ${ }^{11}$ and non-small cell lung cancers. ${ }^{13}$ Furthermore, Rab18 serves as a novel tumor antigen in the screening of childhood medulloblastoma DNA libraries. ${ }^{9}$ Rab18 functions as a promoter of cell proliferation in hepatoma cell lines. ${ }^{10}$ Knockdown of Rab18 suppressed lung cancer cell growth in A549 and H23 cell lines. ${ }^{13}$ However, there has been no report concerning the protein expression of Rab18 in human HNSCCs. In addition, its biological roles and potential mechanism have not been definitively determined.
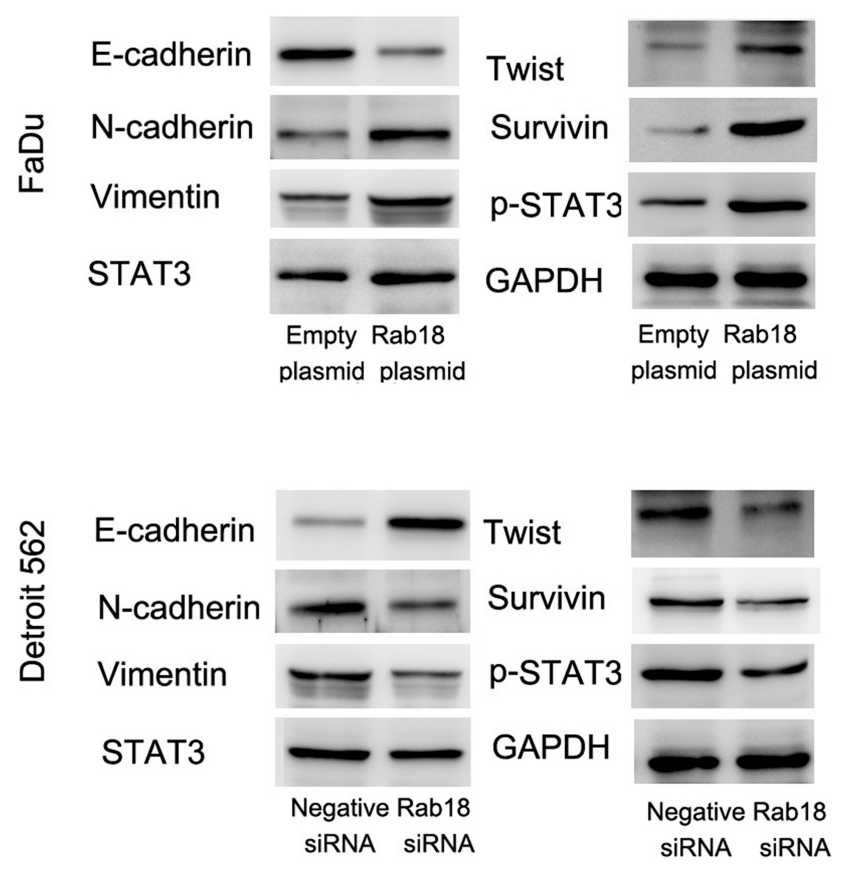

Figure 5 Rab 18 regulates E-cadherin, Twist, Survivin and STAT3 phosphorylation. Western blot showed that RabI 8 overexpression significantly downregulated E-cadherin, while upregulated N-cadherin, Vimentin, Twist, P-STAT3 and Survivin in FaDu cell line. Rabl8 siRNA knockdown in Detroit562 cell line induced E-cadherin expression with inhibition of N-cadherin, Vimentin, Twist, P-STAT3 and Survivin.

Abbreviations: STAT3, signal transducer and activator of transcription 3; p-STAT3, phospho- signal transducer and activator of transcription 3; siRNA, small interfering RNA. 


\section{A}

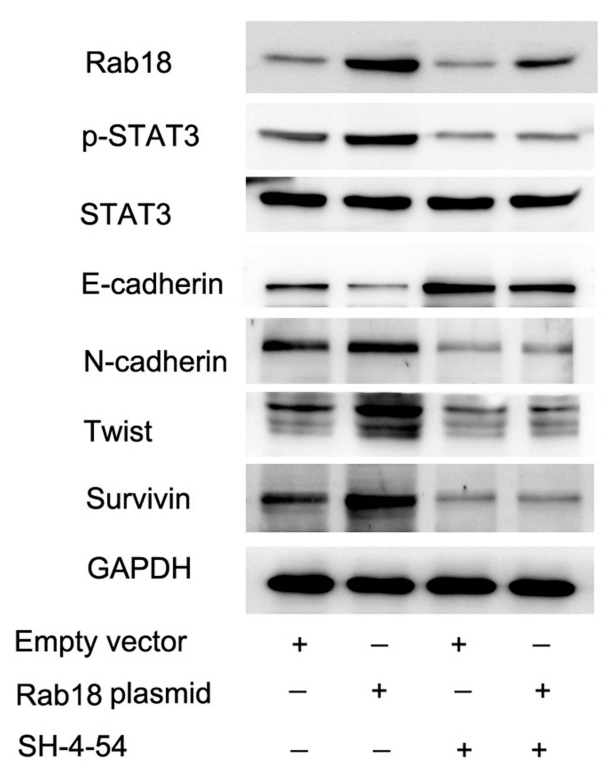

B

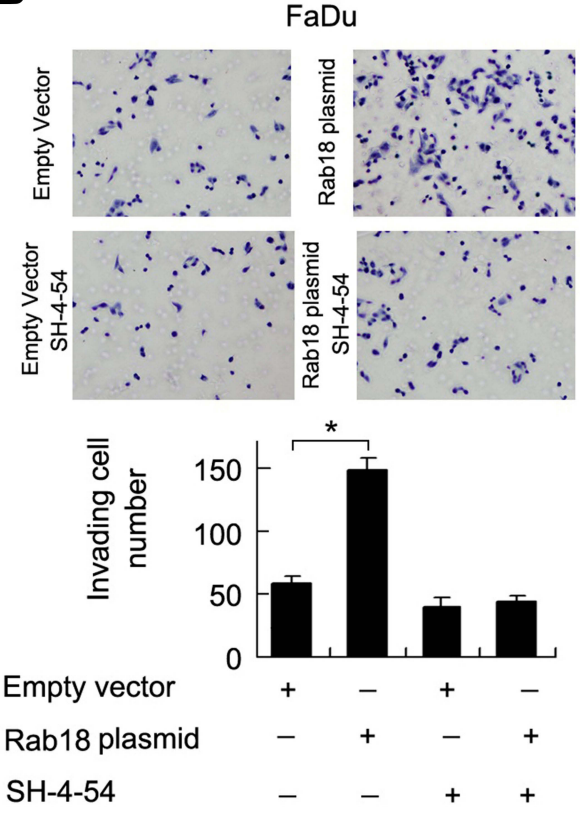

Figure 6 Rabl8 regulates E-cadherin, Survivin and invasion through STAT3 signaling pathway. (A) Western blot showed that STAT3 inhibitor SH-4-54 blocked phosphorylation of STAT3 without change of total STAT3. SH-4-54 treatment upregulated E-cadherin protein and downregulated protein levels of Twist, N-cadherin and Survivin. In cells treated with SH-4-54, the effects of Rabl8 on E-cadherin, Twist, N-cadherin and Survivin were not significant. (B) SH-4-54 treatment led to significant inhibition of cell invasiveness. Rabl8 failed to upregulate FaDu cell invasion in SH-4-54 treated cells. *p $<0.05$.

Abbreviations: STAT3, signal transducer and activator of transcription 3; p-STAT3, phospho- signal transducer and activator of transcription 3; GAPDH, glyceraldehyde 3phosphate dehydrogenase.

To validate the clinical significance of Rab18 in HNSCC, immunohistochemistry was used, showing that compared with normal tissues, Rab18 protein was significantly upregulated in $40.5 \%$ of the HNSCC specimens tested. Rab18 overexpression was associated with lymph node metastasis, higher T stage and higher TNM stage, which was further supported by TCGA and Oncomine data analyses. These data indicated Rab18 was a clinical biomarker of cancer aggressiveness in HNSCC.

We further used Rab18 plasmid and siRNA to validate its biological functions. MTT and colony formation showed that forced expression of Rab18 promoted in vitro cell growth with activated G1-S cell cycle progression, which makes Rab18 as a growth promoter in HNSCC.

The EMT is crucial for the invasion, progression, and metastasis of epithelial carcinogenesis. ${ }^{14,15}$ This process is also associated with tumor cell invasion and metastasis in HNSCC, which lead to poor patient outcome. ${ }^{16}$ E-cadherin is a key component of adherens junction (AJ), which serve as epithelial markers during this process. ${ }^{17}$ In the present study, we showed that Rab18 facilitated HNSCC cell invasion with downregulation of E-cadherin and upregulation of N-cadherin, Vimentin, suggesting that Rab18 was a novel regulator of EMT in HNSCC. In addition, we found upregulation of Twist protein after Rab18 expression. Twist is a helix-loop-helix transcription factor which functions as a regulator of embryonic development and plays important roles during mesenchymal differentiation. ${ }^{18}$ Twist is upregulated in various human tumors including HNSCC and plays an important role during EMT process, ${ }^{18}$ suggesting Rab18 promoted EMT and invasion through Twist upregulation.

During clinical practice, HNSCC often develop resistance to chemotherapy which greatly limits its efficiency. Therefore, we characterized the role and mechanism of Rab18 on cisplatin sensitivity. Rab18 overexpression decreased cisplatin sensitivity and reduced cisplatininduced apoptosis while its depletion showed the opposite effect. Mitochondrial plays a central role in cisplatininduce apoptosis. ${ }^{19-22}$ Loss of mitochondrial membrane potential triggered by treatment increases permeability and releases cytochrome c into cytosol, which activates apoptotic pathways. ${ }^{23}$ Our data showed that Rab18 overexpression could maintain mitochondrial membrane potential during cisplatin treatment, suggesting Rab18 might inhibit apoptosis and reduce cisplatin sensitivity through protection of mitochondrial function. We also found that 
Rab18 upregulated the Survivin protein, which is an antiapoptosis protein capable of blocking loss of $\Delta \psi \mathrm{m}^{24}$ Thus, it is possible that Rab18 prevented loss of $\Delta \psi \mathrm{m}$ through Survivin during cisplatin treatment.

To better understand the connection between Rab18, Survivin and EMT in more detail, we checked several related signaling pathways and found that Rab18 activated STAT3 signaling. The STAT3 transcription factor is an important signaling molecule with a broad variety of biological functions. ${ }^{25}$ STAT3 is constitutively activated in a number of human tumors including HNSCC, which could also be induced by many cytokines and growth factor receptors. ${ }^{26}$ Nuclear localization of STAT3 and mediates the expression of a variety of genes including Twist ${ }^{25,27}$ and Survivin. ${ }^{28,29}$ The STAT3/Twist signaling has been reported to induce gastric cancer cell invasion. ${ }^{30}$ Our results demonstrated that Rab18 was able to activate STAT3 phosphorylation. In addition, SH-4-54, a potent STAT3 inhibitor, blocked the role of Rab18 on Twist, E-cadherin, Survivin and cancer invasion. These findings suggested a link between Rab18, STAT3 and HNSCC invasion/chemo-resistance.

The exact mechanism of Rab18 on STAT3 activation remains unknown. STAT3 is a cytoplasmic transcription factors that translocate into the nucleus and regulate gene expression. While this translocation event is essential for gene regulation by STAT3. It has been reported that transport of STAT3 is an active process that requires receptormediated endocytosis. Because Rab18 is involved in this process, we believed that Rab18 plays a part during STAT3 transportation, although further studies need to be conducted to confirm this possibility.

In conclusion, this study identified a novel role of Rab18 as an oncoprotein overexpressed in human HNSCCs. Our results suggested that the activation of Rab18/STAT3 signaling was associated with proliferation, invasion and chemo-resistance of HNSCC cells. Our findings provided the possibility of targeting the Rab18/STAT3 axis as a potential therapeutic strategy.

\section{Acknowledgment}

The study was supported by The National Natural Science Foundation of China (No. 81802703) to Hong Li.

\section{Disclosure}

The authors report no conflicts of interest in this work.

\section{References}

1. Chen W, Zheng R, Baade PD, et al. Cancer statistics in China, 2015. CA Cancer J Clin. 2016;66(2):115-132. doi:10.3322/caac.21338

2. Siegel RL, Miller KD, Jemal A. Cancer statistics, 2015. CA Cancer J Clin. 2015;65(1):5-29. doi:10.3322/caac.21254

3. Chia WJ, Tang BL. Emerging roles for Rab family GTPases in human cancer. Biochim Biophys Acta. 2009;1795(2):110-116. doi:10.1016/j.bbcan.2008.10.001

4. Cheng KW, Lahad JP, Gray JW, Mills GB. Emerging role of RAB GTPases in cancer and human disease. Cancer Res. 2005;65 (7):2516-2519. doi:10.1158/0008-5472.CAN-05-0573

5. Dong Q, Fu L, Zhao Y, et al. Rab11a promotes proliferation and invasion through regulation of YAP in non-small cell lung cancer. Oncotarget. 2017;8(17):27800-27811. doi:10.18632/oncotarget.15 359

6. Amornphimoltham P, Rechache K, Thompson J, et al. Rab25 regulates invasion and metastasis in head and neck cancer. Clin Cancer Res. 2013;19(6):1375-1388. doi:10.1158/1078-0432.CCR-12-2858

7. Pulido MR, Diaz-Ruiz A, Jimenez-Gomez Y, et al. Rab18 dynamics in adipocytes in relation to lipogenesis, lipolysis and obesity. PLoS One. 2011;6(7):e22931. doi:10.1371/journal.pone.0022931

8. Bem D, Yoshimura S, Nunes-Bastos R, et al. Loss-of-function mutations in RAB18 cause Warburg micro syndrome. Am J Hum Genet. 2011;88(4):499-507. doi:10.1016/j.ajhg.2011.03.012

9. Behrends U, Schneider I, Rossler S, et al. Novel tumor antigens identified by autologous antibody screening of childhood medulloblastoma cDNA libraries. Int $J$ Cancer. 2003;106(2):244-251. doi:10.1002/ijc. 11208

10. You X, Liu F, Zhang T, Li Y, Ye L, Zhang X. Hepatitis B virus X protein upregulates oncogene Rab18 to result in the dysregulation of lipogenesis and proliferation of hepatoma cells. Carcinogenesis. 2013;34(7):1644-1652. doi:10.1093/carcin/bgt089

11. Gong T, Zhou B, Liu M, et al. RAB18 promotes proliferation and metastasis in hepatocellular carcinoma. Am J Transl Res. 2019;11 (2):1009-1019.

12. Wu B, Qi R, Liu X, Qian L, Wu Z. Rab18 overexpression promotes proliferation and chemoresistance through regulation of mitochondrial function in human gastric cancer. Onco Targets Ther. 2018; 11:7805-7820. doi:10.2147/OTT.S170829

13. Zhong K, Chen K, Han L, Li B. MicroRNA-30b/c inhibits non-small cell lung cancer cell proliferation by targeting Rab18. BMC Cancer. 2014;14:703. doi:10.1186/1471-2407-14-703

14. Beuran M, Negoi I, Paun S, et al. The epithelial to mesenchymal transition in pancreatic cancer: a systematic review. Pancreatology. 2015;15(3):217-225. doi:10.1016/j.pan.2015.02.011

15. Katoh M. Epithelial-mesenchymal transition in gastric cancer (Review). Int J Oncol. 2005;27(6):1677-1683.

16. Pectasides E, Rampias T, Sasaki C, et al. Markers of epithelial to mesenchymal transition in association with survival in head and neck squamous cell carcinoma (HNSCC). PLoS One. 2014;9(4):e94273. doi:10.1371/journal.pone.0094273

17. Ma K, Fan Y, Dong X, et al. MTA1 promotes epithelial to mesenchymal transition and metastasis in non-small-cell lung cancer. Oncotarget. 2017;8(24):38825-38840. doi:10.18632/oncotarget.16 404

18. Zeng J, Zhan P, Wu G, et al. Prognostic value of Twist in lung cancer: systematic review and meta-analysis. Transl Lung Cancer Res. 2015;4(3):236-241. doi:10.3978/j.issn.2218-6751.2015.04.06

19. Guerra F, Arbini AA, Moro L. Mitochondria and cancer chemoresistance. Biochim Biophys Acta. 2017;1858(8):686-699. doi:10.1016/j. bbabio.2017.01.012

20. Kim JS, Lee JM, Chwae YJ, et al. Cisplatin-induced apoptosis in Hep3B cells: mitochondria-dependent and -independent pathways. Biochem Pharmacol. 2004;67(8):1459-1468. doi:10.1016/j.bcp.2003. 12.013 
21. Zhao W, You CC, Zhuang JP, et al. Viability inhibition effect of gambogic acid combined with cisplatin on osteosarcoma cells via mitochondria-independent apoptotic pathway. Mol Cell Biochem. 2013;382(1-2):243-252. doi:10.1007/s11010-013-1740-5

22. Li H, Fu L, Liu B, Lin X, Dong Q, Wang E. Ajuba overexpression regulates mitochondrial potential and glucose uptake through YAP/ Bcl-xL/GLUT1 in human gastric cancer. Gene. 2019;693:16-24. doi:10.1016/j.gene.2019.01.018

23. Battogtokh G, Cho YY, Lee JY, Lee HS, Kang HC. Mitochondrialtargeting anticancer agent conjugates and nanocarrier systems for cancer treatment. Front Pharmacol. 2018;9:922. doi:10.3389/ fphar.2018.00922

24. Ausserlechner MJ, Hagenbuchner J. Mitochondrial survivin - an Achilles' heel in cancer chemoresistance. Mol Cell Oncol. 2016;3 (2):e1076589. doi:10.1080/23723556.2015.1076589

25. Li B, Huang C. Regulation of EMT by STAT3 in gastrointestinal cancer (Review). Int J Oncol. 2017;50(3):753-767. doi:10.3892/ ijo. 2017.3846
26. Mali SB. Review of STAT3 (Signal Transducers and Activators of Transcription) in head and neck cancer. Oral Oncol. 2015;51(6):565569. doi:10.1016/j.oraloncology.2015.03.004

27. Cheng GZ, Zhang WZ, Sun M, et al. Twist is transcriptionally induced by activation of STAT3 and mediates STAT3 oncogenic function. J Biol Chem. 2008;283(21):14665-14673. doi:10.1074/jbc. M707429200

28. Scheper MA, Nikitakis NG, Sauk JJ. Survivin is a downstream target and effector of sulindac-sensitive oncogenic Stat3 signalling in head and neck cancer. Int J Oral Maxillofac Surg. 2007;36(7):632-639. doi:10.1016/j.ijom.2007.04.003

29. Gritsko T, Williams A, Turkson J, et al. Persistent activation of stat3 signaling induces survivin gene expression and confers resistance to apoptosis in human breast cancer cells. Clin Cancer Res. 2006;12 (1):11-19. doi:10.1158/1078-0432.CCR-04-1752

30. Hsu KW, Hsieh RH, Huang KH, et al. Activation of the Notch1/ STAT3/Twist signaling axis promotes gastric cancer progression. Carcinogenesis. 2012;33(8):1459-1467. doi:10.1093/carcin/bgs165

\section{Publish your work in this journal}

OncoTargets and Therapy is an international, peer-reviewed, open access journal focusing on the pathological basis of all cancers, potential targets for therapy and treatment protocols employed to improve the management of cancer patients. The journal also focuses on the impact of management programs and new therapeutic agents and protocols on patient perspectives such as quality of life, adherence and satisfaction. The manuscript management system is completely online and includes a very quick and fair peer-review system, which is all easy to use. Visit http://www.dovepress.com/ testimonials.php to read real quotes from published authors. 